\title{
Use of Selective Media for Detection of Cephalothin-Resistant Bacteria in Surgical Patients
}

Sérgio A.C. Melo ${ }^{1}$, Eduardo A.R. Castro ${ }^{2}$, Antônio C. Ponce-de-Leon ${ }^{3}$ and José A.A. Pereira ${ }^{1}$

\author{
Microbiology and Immunology Service, Medical \\ Science School ${ }^{1}$; Committee of Control of Hospital \\ Infections, Pedro Ernesto Universitary Hospital ${ }^{2}$; \\ Institute of Social Medicine ; State University of Rio \\ de Janeiro, Rio de Janeiro, RJ, Brazil
}

\begin{abstract}
Bacterial colonization in hospitalized patients is an important step in nosocomial infections. Frequent employment of antimicrobials can modify patients' normal microbiota, favoring colonization and infection by antimicrobial-resistant microorganisms. First-generation cephalosporins are frequently used as prophylactic antibiotics in surgery. Intestinal, oropharyngeal and skin colonization by cephalothin-resistant microorganisms were studied in 60 pre-operative patients at the Hospital Universitário Pedro Ernesto. Feces were cultured in Eosin-methylene blue medium containing $32 \mu \mathrm{g} / \mathrm{mL}$ of cephalothin. Swabs obtained from the oropharynx and from skin were inoculated in cistein-lactose electrolytes-deficient medium containing $32 \mu \mathrm{g} / \mathrm{mL}$ of cephalothin. Isolated strains were identified and tested for susceptibility to antimicrobials by disk diffusion. Cephalothin-resistant strains were isolated from the feces of 59 patients $(98 \%)$, from the oropharynx of 13 patients $(22 \%)$ and from skin in 10 patients $(17 \%)$. Enterobacter cloacae was predominant in feces (68\% of the patients) and oropharynx $(13 \%)$. Acinetobacter spp. was the most frequent microorganism isolated from the skin $(10 \%)$. Antimicrobial multiresistant strains were isolated from at least one of the sites in 38 patients $(63 \%)$. The employment of selective medium containing antimicrobials is a relatively simple and efficient method, being useful to evaluate microorganisms from hospitalized patients' microbiota that are relevant as potential pathogens in nosocomial infections.
\end{abstract}

Key Words: Selective media, cephalotin resistance, surgical patients.

Bacterial colonization of hospitalized patients is an important step for the occurrence of endogenous nosocomial infections [1-5]. Many studies show that intestinal, oropharyngeal and skin microbiota are important reservoirs of microorganisms potentially able to cause infections in other sites $[1,2,4,6,7]$.

Along the last decades, there has been a significant increase in bacterial antimicrobial resistance, especially

Received on 20 December 2003; revised 14 April 2004. Address for correspondence: Dr. José A. A. Pereira. Faculdade de Ciências Médicas, Universidade do Estado do Rio de Janeiro, UERJ, Departamento de Patologia e Laboratórios, Av. 28 de Setembro, 87 - fundos, 20551-030 Rio de Janeiro, RJ, Brasil. Telefax: (+5521) 2587-6476.E-MAIL: josep@uerj.br

The Brazilian Journal of Infectious Diseases 2004;8(3):190-196 (C) 2004 by The Brazilian Journal of Infectious Diseases and Contexto Publishing. All rights reserved. in nosocomial environments [8,9]. Due to great selective pressure, abusive employment of antimicrobials may cause changes in patients' microbiota and lead to consequent colonization by antimicrobial-resistant microorganisms $[2,9,10]$. Antimicrobial multiresistance can be observed in bacterial strains that are associated with hospital environments, both colonizing [11,12] and causing infections in hospitalized patients $[1,9]$. These situations emphasize the importance of studying antimicrobial resistance patterns in bacteria that are part of the microbiota in different anatomic sites.

Several authors suggest periodical bacteriological cultures to monitor hospitalized patients, establishing predictive conditions for endogenous infections $[1,4,5,13]$. Surveillance of antimicrobial susceptibility focalized in specific units (such as those which maintain critically ill patients and consequently employ wide 
spectrum antimicrobial agents) could be more useful for evaluating antimicrobial resistance patterns than general hospital data, leading to prompt identification of changes in resistance patterns, allowing faster control measures $[10,14,15]$.

Employment of selective medium may be useful for studying nosocomial infections. Selden et al. [4] utilized MacConkey agar with $10 \mu \mathrm{g} / \mathrm{mL}$ of cloranphenicol and $40 \mu \mathrm{g} / \mathrm{mL}$ of ampicillin for establishing the role of colonization in endogenous infections. Hamilton et al. [1] employed a selective medium containing sulphametoxazol-trimethoprim for demonstrating that colonization of the gastrointestinal tract by multiresistant microorganisms preceded bacteremia in $94 \%$ of the patients.

Etiological agents of surgical infections frequently arise from the patients' own microbiota (mainly in digestive tract surgeries), from contact with surgical staff or from the hospital environment. The majority of surgical infections are caused by components of the microbiota from surgical sites or adjacent areas, with a smaller proportion of exogenous microbiota [7]. In addition, distant sites' microbiota may be a source of surgical infections [16]. Urinary, skin, and respiratory infections are very commonly associated with an increase in surgical infection rates [7]. During the last decade, there have been important changes in bacterial susceptibility related to surgical infections $[8,17]$. Moreover, there has been a strong increase in surgical infections caused by vancomycin-resistant enterococci [17].

Selection of prophylactic antimicrobials must be established mainly based on their efficacy against exogenous and endogenous microorganisms most often isolated from infections in each clinical situation $[6,7,18]$. The main risks of antimicrobial prophylaxis are selective pressure exercised by chosen drug and modifications in normal microbiota, with consequent development of resistant microorganisms. According to Blech [19], antimicrobial prophylaxis could favor quantitative and qualitative changes in the hospital ward ecosystem and in hospital microbiota. Archer [20] observed that Staphylococcus spp. isolated from patients' skin after cardiac surgery were more antimicrobial-resistant than those isolated from the same sites during the pre-operative period, suggesting selection of resistant strains or their acquisition from a nosocomial environment.

Various authors defend pre-operative screening by microbiological surveillance methods to better study antimicrobial prophylaxis outcomes in patients' microbiota, thus to diminish the risks of surgical infections [5,6].

We examine microbial frequency and diversity in intestinal, oropharyngeal and skin colonization of preoperative hospitalized patients by bacteria that are resistant to cephalothin, employing two selective media containing cephalothin. This antimicrobial is used worldwide (including in our hospital) as part of a protocol for surgical prophylaxis, due mainly to its wide spectrum activity against the most frequent causative agents of surgical infections [21]. Moreover, we evaluated cephalothin as a marker to detect resistant and multiresistant strains that are able to colonize hospitalized patients and, eventually, could be etiological agents of surgical infections.

\section{Materials and Methods}

\section{Patient selection and sample acquisition}

Sixty pre-operative adult patients ( 31 female, 29 male) hospitalized at HUPE (a urban general tertiarycare hospital, with 600 beds) were studied from January to May 1997. Twenty-three patients were in the General Surgery Ward, 13 in the Thoracic Surgery Ward, 15 patients were from the Cardiology Ward, and 9 patients were hospitalized in other wards. The patients were selected randomly by sending feces samples from surgical wards as a pre-operative routine procedure. Patients were localized in the surgical wards and skin and oropharynx samples were obtained. Oropharynx specimens were collected with sterile swabs. Skin specimens were collected with sterile swabs, moistened in sterile $0.85 \%$ saline, rubbed on the anterior surface of the arm and the forearm. 
Epidemiological data on the patients, such as age, period of hospitalization, eventual previous hospitalization, prior antimicrobial use, underlying disease and occurrence of surgical or other infections were obtained from medical files, bacteriology laboratory records and interviews.

\section{Sample culture}

Eosin-methylene blue (EMB) medium (Difco Becton Dickinson and Company. Sparks, MD, USA) containing $32 \mu \mathrm{g} / \mathrm{mL}$ of cephalothin (EMB-cephalothin medium) was employed as a selective medium for culturing feces specimens. For skin and oropharynx specimens, CLED (Cistein-Lactose ElectrolytesDeficient) medium (Difco) containing $32 \mu \mathrm{g} / \mathrm{mL}$ of cephalothin (CLED-cephalothin medium) was also used as a selective medium. That concentration corresponds to the lower level of minimal inhibitory concentration to bacterial strains ranked as cephalothinresistant [22]. Selective medium were tested by culturing standard strains. Pseudomonas aeruginosa ATCC 27853 and Klebsiella pneumoniae T401F10 (a strain isolated from feces in a previous study [11], which carries a antimicrobial multiresistance plasmid) are resistant to the cephalothin concentration that we employed. Staphylococcus aureus ATCC 25923 and E. coli K12R23 (a laboratorial strain without a resistance plasmid, employed in a previous study [11]) showed susceptibility to the cephalothin concentration that was employed $(32 \mu \mathrm{g} / \mathrm{mL})$.

Results were observed after 24, 48 and 72 hours of incubation. After this period, specimens with no bacterial growth were considered as negative. Bacterial colonies grown in EMB-cephalothin and CLEDcephalothin media were selected by their morphological features, including all observed bacterial variety. Every morphological type was submitted to Gram's method for the definition of biochemical tests employed to identify bacterial species (or genera).

Catalase-positive Gram-positive cocci were identified according to Kloos and Bannerman [23]. Catalase-negative cocci were submitted to identification tests according to Facklam and Sahm [24].
Enterobacteriaceae were identified according to Edwards and Ewing [25], Suassuna and Suassuna [26] and Farmer III [27]. Non-fermentative Gram-negative bacilli were submitted to identification tests according to Gilligan [28] and von Graevenitz [29]. When bacterial identification was not conclusive, employing only conventional biochemical tests, an automated system was used (VITEK; BioMériux Inc. Hazelwood, Missouri, USA).

We employed the disk diffusion method [30] for characterizing resistance patterns of the strains isolated from selective media. Amikacin $(30 \mu \mathrm{g} / \mathrm{mL})$, ampicillin $(10 \mu \mathrm{g} / \mathrm{mL})$, cephalothin $(30 \mu \mathrm{g} / \mathrm{mL})$, cefoxitin $(30 \mu \mathrm{g} /$ $\mathrm{mL})$, ceftazidime $(30 \mu \mathrm{g} / \mathrm{mL})$, chloramphenicol $(30 \mu \mathrm{g} /$ $\mathrm{mL})$, gentamicin $(10 \mu \mathrm{g} / \mathrm{mL})$, perfloxacin $(5 \mu \mathrm{g} / \mathrm{mL})$, tetracycline $(30 \mu \mathrm{g} / \mathrm{mL})$ and trimethoprimsulfamethoxazole $(1.25 / 23.75 \mu \mathrm{g} / \mathrm{mL})$ (Difco) were tested for Gram-negative bacilli. Additionally, vancomycin $(30 \mu \mathrm{g} / \mathrm{mL})$ and oxacillin $(1 \mu \mathrm{g} / \mathrm{mL})$ (Difco) were tested for staphylococci, and vancomycin for enterococci. Antimicrobial disks were tested with $E$. coli ATCC 25922, Pseudomonas aeruginosa ATCC 27583 and Staphylococcus aureus ATCC 25923. Results were interpreted according to Ferraro et al. [30]. When strains isolated from selective media were cephalothin-sensitive by the disk diffusion method, they were excluded from this study. We considered as multiresistant to antimicrobials those strains showing resistance markers for more than two antimicrobials from different classes.

\section{Results}

A total of 143 bacterial strains were isolated from colonization of the 60 pre-operative patients: 114 strains were isolated from feces $(80 \%), 16(11 \%)$ were isolated from the oropharynx and 13 strains (9\%) were isolated from skin. Cephalothin-resistant strains were isolated from feces of 59 patients $(98 \%)$, from the oropharynx of 13 patients $(22 \%)$ and from skin of 10 patients (17\%). Patients' ages varied from 24 to 87 years $($ median $=54$; average $=53.7)$. Gallbladder lithiasis was the main motive for surgery (14 cases). 
The hospitalization period varied from 1 to 72 days ( median $=7$ days and average $=10.2$ days). Thirteen patients had been hospitalized previously and 9 patients were submitted to antimicrobials in the 6 months before sample collection. There were post-operative infections in 5 patients ( 3 surgical infections, 1 urinary infection and 1 bacteremia).

Enterobacter cloacae was the most frequent microorganism isolated from feces (41 patients, 68\%) and from the oropharynx (8 patients, $13 \%$ ). In the skin, Acinetobacter spp. were predominant, occurring in 6 patients (10\%).

In four cases, strains of the same species showing similar resistance patterns were detected at different anatomic sites in the same patient. Moreover, one patient had a surgical infection caused by Morganella morganii, with resistance patterns similar to a strain of the same species isolated from fecal colonization (data obtained from medical files).

Table 1 shows the frequencies of pre-operative patients colonized in feces, the oropharynx and skin by cephalothin-resistant categories of bacteria, exclusively or in association with other bacteria.

Antimicrobial multiresistant strains were isolated from at least one of the sites in 38 patients $(63.3 \%)$. Table 2 shows the most frequently observed multiresistance patterns more frequently observed in colonization from feces, oropharynx and skin of preoperative patients.

\section{Discussion}

CLED medium was employed because it allows growth of various taxonomic groups of bacteria that are important in the colonization of hospitalized patients and in the origin of nosocomial infections, including Enterobacteriaceae, and non glucose fermenting Gram-negative bacilii, and Gram-positive cocci, such as Staphylococcus and Enterococcus. EMB medium was employed due to its selectivity for Gram-negative bacilii, which are a considerable fraction of the components of intestinal microbiota [31], and are important agents of extra-intestinal nosocomial infections [4,32]. EMB also allowed the isolation of Enterococcus spp., despite the reduced size of the colonies. However, other media could be useful for this kind of analysis, such as Blood Agar, a selective media for Gram-positive bacteria (Mannitol-oxacillin agar for Staphylococcus spp., for example), or other media for Gram-negative bacteria, such as MacConkey agar.

For characterization of the resistance patterns of strains isolated in selective media, antimicrobials were selected so as to include different chemical groups, facilitating analysis about the multiresistance patterns of bacterial strains. The finding of $63 \%$ of patients colonized by multiresistant strains that are relevant as potential agents of surgical infections in at least one of the analyzed sites suggests that cephalothin is useful for the detection of multiresistant strains.

Occurrence of strains of the same species showing similar resistance patterns at different anatomic sites in the same patient could be due to a common source and/or to bacterial translocation [16]. Moreover, we found fecal colonization and surgical infection by strains of the same bacterial species showing similar resistance patterns, in the same patient. These findings suggest progressive establishment of several potential sources for surgical infections, possibly favored by prolonged hospitalization and/or by antimicrobial selective processes [7,10,33,34].

Lorian [35] indicated that there was inadequate accessibility of data about bacterial antimicrobial susceptibility, emphasizing the almost inexistence of national and international data concerning this topic. We believe in the necessity of periodic surveys, focusing on antimicrobial resistance of the microorganisms most frequently implicated in colonization and infection in hospitalized patients, leading to a more suitable choice of antimicrobials for therapeutic and prophylactic use. In our study, cephalothin-resistant bacterial species and genera isolated from intestinal, oropharyngeal and skin colonization in pre-operative patients corresponded, in part, to those bacterial species and genera isolated from cases of infection in surgical patients in our hospital (Staphylococcus aureus, Escherichia coli, Enterococcus sp). We can not affirm that those strains 
Table 1. Number of patients (out of 60) from which cephalothin-resistant bacteria were isolated from feces, oropharynx and skin in surgical patients in HUPE-UERJ in 1997.

\begin{tabular}{|c|c|c|c|}
\hline Microorganism category & Feces & Oropharynx & Skin \\
\hline Enterobacteriaceae & 33 & 8 & 1 \\
\hline Enterococci & 4 & 0 & 0 \\
\hline Non-fermenters GNB* & 2 & 3 & 3 \\
\hline Staphylococci** & 0 & 1 & 5 \\
\hline Enterobacteriaceae and non-fermenters GNB $* \dagger$ & 8 & 1 & 1 \\
\hline Enterobacteriaceae and enterococci ${ }^{\dagger}$ & 8 & 0 & 0 \\
\hline Enterococci and non-fermenters GNB $* \dagger$ & 2 & 0 & 0 \\
\hline $\begin{array}{l}\text { Enterobactericeae, enterococci and } \\
\text { non-fermenters GNB }\end{array}$ & 1 & 0 & 0 \\
\hline $\begin{array}{l}\text { Enterobacteriaceae, staphylococci** and } \\
\text { non-fermenters GNB }{ }^{*}\end{array}$ & 1 & $\begin{array}{c}0 \\
47\end{array}$ & $\begin{array}{c}0 \\
50\end{array}$ \\
\hline
\end{tabular}

*Non-fermenters: glucose non-fermenters; GNB: Gram-negative bacilli (Pseudomonas mendocina, Pseudomonas aeruginosa, Pseudomonas alcaligenes, Acinetobacter spp., and Stenotrophomonas maltophilia).

** Coagulase-negative Staphylococcus and Staphylococcus aureus.

${ }^{\dagger}$ Two or more groups of microorganisms colonizing the same patient.

Table 2. Multiresistance patterns most frequently observed in cephalothin-resistant bacteria isolated from fecal, oropharyngeal and skin colonization in 60 pre-operative patients at HUPE(1997).

\begin{tabular}{llccr}
\hline Resistance patterns & Species & \multicolumn{2}{c}{ Number of patients } \\
\cline { 3 - 5 } & & Feces & Oropharynx & Skin \\
\hline Ami-Amp-Cef-Cfo- & Staphylococcus spp. & 1 & $-*$ & 1 \\
Caz-Gen-Oxa-Pef-Sul & & & & \\
Amp-Cef-Cfo-Caz- & Staphylococcus spp. & - & - & 1 \\
Clo-Gen-Oxa-Sul-Tet & Staphylococcus aureus & - & - & 1 \\
Ami-Amp-Cef-Cfo-Caz- & Enterococcus spp. & 4 & - & - \\
Clo-Gen-Oxa-Pef-Sul & Staphylococcus spp. & - & 1 & - \\
Ami-Amp-Cef-Cfo-Caz- & Pseudomonas mendocina & 1 & - & - \\
Clo-Gen-Pef-Sul-Tet & Enterococcus spp. & 3 & - & - \\
& Acinetobacter spp. & - & 1 & - \\
\hline
\end{tabular}

Ami-amikacin; Amp- ampicilin; Cef- cephalothin; Cfo-cefoxitin; Caz- ceftazidime; Clo-chloranphenicol; Gengentamicin; Oxa- oxacilin; Pef- pefloxacin; Sul- trimethoprim-sulfametoxazole; Tet- tetracycline. ${ }^{*}=$ not detected. 
are the same strains involved in surgical infections. Such a possibility could only be investigated by molecular techniques.

Despite worldwide employment of cephalothin in pre-operative prophylaxis, due to its efficacy on the main agents of surgical infections, such as Staphylococcus aureus and E. coli [7,21], some potential etiological agents have natural or acquired cephalothin-resistance, such as Enterobacter spp. and Enterococcus spp. [36] and/or are multiresistant. This fact could compromise cephalothin's use. However, the frequencies of these causative agents are probably small. Also, part of the surgical infections in these patients could be from exogenous sources [18,21]. Moreover, a portion of surgical infectious agents is cephalothin-susceptible, and not all cephalothinresistant bacteria are relevant as etiological agents of surgical infections [8,21].

It is our point of view that bacterial colonization in hospitalized patients must be monitored during their internment, examining which microorganisms colonize particular sites, their resistance patterns, and how this resistance could interfere in therapeutic and prophylactic procedures. This surveillance should preferably be executed in specific units, since the spectrum of microorganisms and bacterial resistance can vary from unit to unit.

We think that the evaluation of nosocomial colonization employing selective media containing antimicrobials is a relatively simple and efficient method, which could be useful for knowledge on microorganisms that are part of hospitalized patients' microbiota, and which are relevant as potential pathogens in nosocomial infections. The selective media that we employed allowed us to detect some potential surgical infectious agents (endogenous, cephalothin-resistant or multiresistant, and relevant surgical infectious agents). Consequently, we suggest the employment of selective media containing antimicrobials (especially, those used in the prophylaxis of surgical infection) as laboratory routine for helping choose antimicrobials employed in therapeutic and prophylactic regimens (especially for some groups, such as extremely aged, transplanted and neoplastic patients) and for aiding in studies about outbreaks (such as ESBL and VRE outbreaks), under Nosocomial Infections Control Committee's supervision.

In addition, the importance of measures looking for preventing cross colonization in hospitalized patients, especially by antimicrobial multiresistant microorganisms, must be emphasized. Reduction of hospital stay, inclusively in pre-operative periods, careful hand-washing and use of barriers, such as gloves and masks, by hospital staff, and isolation of patients colonized and/or infected with multiresistant bacteria are indispensable measures to attain nosocomial infections control.

\section{References}

1. Hamilton D.J., Ulness B.K., Baugher L.K., Counts G.W. Comparison of a novel trimethoprim-sulfamethoxazole containing medium (XT 80) with kanamycin agar for isolation of antibiotic-resistant organisms from stool and rectal cultures of marrow transplant patients. J Clin Microbiol 1987;25(10):1886-90.

2. Johanson Jr., W.G., Pierce A.K., Sanford J.P., Thomas G.D. Nosocomial respiratory infections with Gram-negative bacilli. The significance of colonization of the respiratory tract. Ann Intern Med 1972;77: 701-6.

3. Manson W.L., Klasen H.J., Sauer E.W., Olieman A. Selective intestinal decontamination for prevention of wound colonization in severely burned patients: a retrospective analysis. Burns 1992;18(2):98-102.

4. Selden R., Lee S., Wang W.L.L., et al. Nosocomial Klebsiella infections: intestinal colonization as reservoir. Ann Intern Med 1971;74:657-64.

5. Tanzer M., Miller J., Richards G.K. Preoperative assessment of skin colonization and antibiotic effectiveness in total knee arthroplasty. Clin Orthop 1994;229:163-8.

6. Nichols R.L. Surgical wound infection. Am J Med 1991;91:3B-54S.

7. Wong E.S. Surgical site infections.In: Mayhall, C.G.(ed). Hospital Epidemiology and Infection Control. Williams \& Wilkins, Baltimore, 1996, p.154-74.

8. Neu H.C. Emerging trends in antimicrobial resistance in surgical infections. A review. Eur J Surg 1994;573:7-18.

9. Toltzis P., Blumer J.L. Antibiotic-resistant Gram-negative bacteria in the critical care setting. Pediatr Clin North Am 1995;42 (3):687-702.

10. Jones R.N. The current and future impact of antimicrobial resistance among nosocomial bacterial pathogens. Diagn Microbiol Infect Dis 1992; 15:35-105. 
11. Melo S.A.C., Castro E.A.R., Pereira J.A.A., Suassuna I. Use of a selective medium with potassium tellurite to follow intestinal colonization of hospitalized patients by drug-resistance Enterobacteriaceae. Mem Inst Oswaldo Cruz 1993;88(1):135-40.

12. Vieira L.A., Castro E.A.R., Duarte J.L.B., et al. Colonização intestinal de recém-nascidos por enterobactérias multirresistentes a antimicrobianos em unidade neonatal. J Pediatr (RJ) 1999;75:83-90.

13. Schimpff S.C., Young V.M., Greene W.H.,et al. Origin of infection in acute nonlymphocytic leukemia. Significance of hospital acquisition of potential pathogens. Ann Intern Med 1972;77:708-14.

14. Bryce E.A., Smith J.A. Focused microbiological surveillance and Gram-negative beta-lactamasemediated resistance in a intensive care unit. Infect Control Hosp Epidemiol 1994;15 (7):447-55.

15. Koontz F.P. Microbial resistance surveillamce techniques. Blood culture versus multiple body site monitoring. Diagn Microbiol Infect Dis 1992;15(2 Suppl):31S-5S.

16. Wiley A.M., Ha' eri G.B. Routes of infection. A study of using " tracer particles " in the orthopedic operating room. Clin Orthop 1979;139:150-5.

17. De Vera M.E., Simmons R.L. Antibiotic-resistant enterococci and the changing face of surgical infections. Arch Surg 1996;131(3):338-42.

18. Drugeon H.B. Criteres microbiologiques du choise d'un antibiotique pour l' antibioprophylaxie en chirurgie. Ann Fr Anesth Remain 1994;13(5 Suppl):525-33.

19. Blech M.F. Impact de l' antibioprophylaxie sur l' ecologie microbienne. Ann Fr Anesth Remain 1994;13(5 Suppl):545-50.

20. Archer G.L. Alterations of cutaneous staphylococcal flora as a consequence of antimicrobial prophylaxis. Rev Infect Dis 1991; 13 (Suppl 10):S 805-9.

21. Rabhae G.N., Ribeiro Filho N., Fernandes A.T. Infecção do sítio cirúrgico. In: Fernandes A.T.(ed). Infecção Hospitalar e suas Interfaces na Área de Saúde. São Paulo, Atheneu, 2000, p.479-505.

22. Woods G.L., Washington J.A. Antimicrobial susceptibility tests: dilution and disk diffusion methods. In: Murray P.R.(Ed in Chief), Baron E.J., Pfaller M.A., et al. Manual of Clinical Microbiology. ASM Press, Washington, 1995, p.1327-41.

23. Kloos W.E., Jorgensen J.H. Staphylococci. In: Murray P.R.(Ed in Chief), Baron E.J., Pfaller M.A., et al. Manual of Clinical Microbiology. ASM Press, Washington, 1995, p.282-98.

24. Facklam R.R., Sahm D.F. Enterococcus. In: Murray P.R.(Ed in Chief), Baron E.J., Pfaller M.A., et al. Manual of Clinical Microbiology, ASM Press, Washington, 1995, p.308-14.
25. Edwards P.R., Ewing W.H. Identification of Enterobacteriaceae. Burgess Publishing Co., Minneapolis, 1972, p.21-47.

26. Suassuna I., Suassuna I.R. Duplo açúcar uréia (DAU), um meio de triagem para enterobactérias. Rev Bras Patol Clin 1978; 14(4):201-203.

27. Farmer III J.J. Enterobacteriaceae: Introduction and Identification. In: Murray P.R.(Ed in Chief), Baron E.J., Pfaller M.A., et al. Manual of Clinical Microbiology. ASM Press, Washington, 1995, p.438-49.

28. Gilligan P.H. Pseudomonas and Burkholderia. In: Murray P.R.(Ed in Chief); Baron E.J., Pfaller M.A.,et al. Manual of Clinical Microbiology. ASM Press, Washington, 1995, p.509-19.

29. Von Graevenitz A. Acinetobacter, Alcaligenes, Moraxella and other nonfermentative Gram-negative bacteria. In: Murray P.R.(Ed in Chief), Baron E.J., Pfaller M.A., et al. Manual of Clinical Microbiology. ASM Press, Washington, 1995, p.520-32.

30. Ferraro M.J., Craig W.A., Dudley M.N., et al. Performance standards for Antimicrobial Disk Susceptibility test; approved standard. Wayne, USA: NCCLS, 2000.

31. Pelczar Jr. M.J., Chan E.C.S., Krieg N.R. Microbiology: concepts and applications. Mc Graw-Hill, New York, 1993.

32. Luiten E.J., Hop W.C., Lange J.F., Bruinig H.A. Controlled clinical trial of selective decontamination for the treatment of severe acute pancreatitis. Ann Surg 1995;222(1):57-65.

33. Arnow P.M., Flaherty J.P. Nonfermentative Gram-negative bacilli. In: Mayhall CG.(ed.) Hospital Epidemiology and Infection Control. Baltimore, Williams \& Wilkins, 1996, p.366-387.

34. George D.L. Nosocomial pneumonia. In: Mayhall C.G. (ed). Hospital Epidemiology and Infection Control. Williams \& Wilkins, Baltimore, 1996, p.175-195.

35. Lorian V. The need for surveillance techniques. Infect Control Hosp. Epidemiol 1995;16(11):638-41. 